\title{
Vorwort - conexus 1/2018: Lust und Leiden
}

Rother, Wolfgang ; Baer, Josette

DOI: https://doi.org/10.24445/conexus.2018.01.004

Posted at the Zurich Open Repository and Archive, University of Zurich ZORA URL: https://doi.org/10.5167/uzh-160061

Journal Article

Published Version

\section{(c) $(1) \odot$}

The following work is licensed under a Creative Commons: Attribution-NonCommercial-NoDerivatives 4.0 International (CC BY-NC-ND 4.0) License.

Originally published at:

Rother, Wolfgang; Baer, Josette (2018). Vorwort - conexus 1/2018: Lust und Leiden. conexus, (1):24-28.

DOI: https://doi.org/10.24445/conexus.2018.01.004 


\section{Wolfgang Rother und Josette Baer Vorwort}

Dass wir die Lust dem Leiden vorziehen, dass wir von Natur aus nach Lust streben und Schmerzen nach Möglichkeit zu vermeiden suchen, gilt als anthropologischer Gemeinplatz. Doch allem Anschein und unserer Intuition zum Trotz sind Lust und Leiden nicht unbedingt konträre Empfindungen oder kontradiktorische Begriffe. Die Wahrheit ist - wie so oft bei einfachen und unbestrittenen Dingen - komplexer und komplizierter. Wir gehen bekanntlich solchen Lüsten aus dem Weg, an deren Konsequenzen wir möglicherweise leiden, und sind umgekehrt ohne weiteres bereit, um späterer Lustempfindungen oder auch nur um der Schmerzvermeidung willen Schmerzen zu ertragen. Religiöse Menschen fasten, bussfertige Ordensleute geisseln sich, Priester und Stoiker üben sich in strenger Enthaltsamkeit - und sind glücklich. Marathonläuferinnen, Jogger und Kraftsportler quälen ihren Körper und fühlen sich gut dabei. Masochisten empfinden Lust, wenn ihnen Schmerzen zugefügt werden.

Nicht Lust oder Leiden, sondern Lust und Leiden: Die vorliegende erste Nummer von conexus enthält Aufsätze, die das Thema aus literaturwissenschaftlicher und kunstgeschichtlicher, historischer und politischer, kulturanthropologischer und medizinischer Perspektive beleuchten. Vorangestellt ist eine kurze Einleitung, in der Wolfgang Rother den Versuch einer ontologischen Bestimmung von Lust und Leiden und ihres Verhältnisses zueinander unternimmt.

Den Auftakt bildet Ulrike Zeuchs einfühlsame biographischphilologische Lektüre von Goethes Trilogie der Leidenschaft (1827), 
in der die dichterische Verarbeitung von Leiderfahrungen als mögliche Strategie ihrer Bewältigung untersucht wird. Wegleitend sind dabei die in Psychoanalyse und Literaturwissenschaft diskutierten Fragen, wie ein Mensch in der Retrospektive ein angemessenes Verhältnis zu eigenem Leid entwickeln kann, ob und wie es Goethe gelingt, durch die literarische Verarbeitung von persönlichen Enttäuschungen und Verletzungen $\mathrm{zu}$ einer versöhnlich-souveränen Haltung zur eigenen Vergangenheit zu gelangen, welches die adäquate Form der Darstellung von Leid ist, und inwiefern durch literarische Verdichtung Individuelles und Persönliches transzendiert und als Allgemeines mitteilbar gemacht werden kann.

Einen ähnlichen Ansatz verfolgt Barbara von Orelli-Messerli, die die Darstellung von Leiden und Lust im künstlerischen Werk von Otto Dix untersucht. Exemplarisch für den Blick der Neuen Sachlichkeit sind drei Gemälde von Otto Dix, in denen das Leiden der Menschen im Ersten Weltkrieg thematisiert wird: die beiden Gemälde Kriegskrüppel (1920) und Schützengraben (1923), die schon in der Weimarer Zeit als antipatriotisch und dann von den Nationalsozialisten als wehrkraftzersetzend diffamiert wurden und seit der Münchener Ausstellung Entartete Kunst von 1937 verschollen sind, sowie das Triptychon Der Krieg (1929/1932), das Otto Dix vor der Zerstörung durch die Nazis retten konnte. In einem Exkurs, der das Motiv des Krieges und des Leidens aufgreift, werden die Nietzsche-Darstellungen von Otto Dix diskutiert: die Gipsbüste des Philosophen (1912) und die Lithographie Der Gekreuzigte (Nietzsche) (1969). Beispielhaft für die Darstellung der Lust ist die Grossstadt (1927/1928), ein Triptychon, das die gesellschafts- und kulturkritische Haltung des Künstlers zu den sogenannten Goldenen Zwanziger Jahren zum Ausdruck bringt. Die Ambivalenz der Lust, ihr Kippen in Leiden, stellt Otto Dix im Bildnis der Tänzerin Anita Berber (1925) dar.

An den Ersten Weltkrieg schliessen sich motivisch die Kriege des französischen Kolonialimperialismus an. Den harten Kern der französischen Streitkräfte bildeten die Söldner der Fremdenlegion, deren Leiden und «Lust» in ihren unterschiedlichen Facetten 
Christian Koller aus einer auf Selbstzeugnissen basierenden Alltags- und Innenperspektive untersucht. In Bezug auf das Leiden waren die Legionäre Opfer und Täter. Einerseits wurden sie schikaniert, unterlagen einem drakonischen Disziplinar- und Strafsystem und litten nicht nur physisch, sondern auch psychisch - die meisten Legionäre erlebten depressive Anfälle. Andererseits waren sie als Täter in Folter, Massaker und andere Grausamkeiten des Imperialkrieges involviert und fügten anderen Menschen schweres Leid zu. In Bezug auf die Lust der Legionäre werden ihre erotischen und sexuellen Beziehungen untersucht: die oft stabilen, eheähnlichen homosexuellen Verbindungen, die Frequentierung von Truppenbordellen und nordafrikanischen Bordellstädten und schliesslich die Liebesbeziehungen zu einheimischen Frauen.

Ein Klassiker der Weltliteratur steht im Mittelpunkt des Aufsatzes von Erich Bryner. Er bietet eine feinsinnige motivische Rekonstruktion des Themas «Lust und Leiden» in Tolstojs Anna Karenina: das Leiden Annas in und an ihrer unglücklichen Ehe mit einem wenig empathischen, konservativen und karrierefixierten Angehörigen der Sankt Petersburger Grossbourgeoisie, ihr kurzes, aber von Anfang an fragiles und von zahlreichen Konflikten und Spannungen, tiefgreifenden Krisen und bitteren Leiderfahrungen durchzogenes Glück in der Liebesaffäre mit Graf Vronskij und schliesslich ihr verzweifelter Suizid.

Der Beitrag von Josette Baer führt uns in den Alltag der sozialistischen Tschechoslowakei zwischen 1945 und 1989. In einer kultur- und sozialgeschichtlichen, mit zahlreichen Abbildungen illustrierten Studie geht die Autorin den vielfältigen politischen Einflüssen auf die Mode, das Frauenbild und den life style in den Epochen des Stalinismus, der Entstalinisierung und der Zeit nach dem Prager Frühling nach. Mode war im Kalten Krieg stets auch ein identitätsstiftendes Moment des politisch-sozialen Systems. In der systemvergleichend angelegten Studie werden am Leitfaden der amerikanischen Modezeitschrift Vogue auf der einen Seite und tschechoslowakischen Frauenzeitschriften und Werbeplakaten auf der anderen Seite die Entwicklungen der Mode unter kapitalistisch- 
marktwirtschaftlichen und staatlich-planwirtschaftlichen Bedingungen dargestellt und untersucht.

Vom modernen europäischen in den vormodernen asiatischen Osten: Marc Winter sichtet die altchinesische philosophische und erotische Literatur auf die Okkurenz als sadistisch oder masochistisch zu deutender sexueller Konzepte oder Praktiken und sucht nach einer kulturhistorischen Erklärung dafür, warum - zumal angesichts eines jenseits moralischer Kategorien geführten Sexualdiskurses - der Sadomasochismus im vormodernen China, auch wenn er gelegentlich praktiziert worden $\mathrm{zu}$ sein scheint, keine signifikante sexuelle Vorliebe war. Allerdings kannte das alte China durchaus die Verbindung von Lust und Schmerz, und zwar in der konfuzianischen Kindespietät, die in der Kaiserzeit als höchste Tugend galt und in teilweise bizarren Geschichten exemplifiziert wurde.

Den Abschluss bildet ein urologischer Beitrag von Hubert John und Philipp Maletzki über das Leiden von Frauen und Männern an den tabuisierten und von komplexen Schamgefühlen begleiteten Erkrankungen an Harninkontinenz und erektiler Dysfunktion, in dem die unterschiedlichen - konservativen, medikamentösen, apparativen und operativen - Therapiemethoden diskutiert werden.

Am Anfang dieser ersten Nummer von conexus stand die letzte Näheres zu den Umständen im Editorial - in dieser Form veranstaltete Ringvorlesung der Privatdozentinnen und Privatdozenten, Titularprofessorinnen und Titularprofessoren der Universität Zürich, die im Herbstsemester 2017 unter dem Titel «Lust und Leiden» unter der organisatorischen Ägide von Josette Baer durchgeführt wurde. Die vorliegenden Beiträge wurden von Fachleuten in einem anonymen Peer-Review-Verfahren begutachtet und von Wolfgang Rother editorisch betreut, lektoriert und redaktionell bearbeitet.

An dieser Stelle seien diejenigen Vorlesungen erwähnt, die die Kolleginnen und Kollegen aus unterschiedlichen Gründen nicht publizieren wollten. Es waren dies die Vorträge der Medizinhistorikerin Iris Ritzmann, «Wenn die Lust zum Leid wird - medizin- 
historische Überlegungen zu Pathologisierung von Sexualität», des Gastroenterologen Stephan Vavricka, «Fasten: die Grenzerfahrung zwischen Leiden und Lust», des Schmerzmediziners Eli Alon, «Mit Lust gegen das Leiden: Cannabis für die Schmerzbehandlung», und des Theologen und Kirchenhistorikers Jan-Andrea Bernhard, «Auftrag und Selbstverständnis erweckter Pietistinnen. Leiden für das Evangelium und Lust in Gott». Letztere Vorlesung musste wegen Krankheit leider ausfallen. In die Bresche sprang Wolfgang Rother mit einem kurzfristig aus bereits andernorts veröffentlichten Gedanken zusammengestellten Vortrag zu «Lust, Leiden, Schmerz, Krankheit und Tod. Existenzphilosophische Perspektiven».

Zum Schluss ein ganz herzlicher Dank: Er gilt den Kolleginnen und Kollegen für ihre Mitwirkung an der Ringvorlesung und die Überarbeitung ihrer Texte für die Publikation, dem Leiter Open Access der Hauptbibliothek der Universität Zürich, Christian Fuhrer, für die kompetente Beratung bei der Gründung der Zeitschrift conexus, dem Team der Plattform HOPE (Hauptbibliothek Open Access Environment), insbesondere André Hoffmann für den professionellen technischen Support und Margit Dellatorre für die nützlichen Empfehlungen zur Gestaltung der Metadaten, und dem Dekanat der Philosophischen Fakultät und der Hochschulstiftung der Universität Zürich sowie der Privatdozenten-Stiftung der Universität Zürich für die grosszügige Finanzierung der Publikationsaufwände.

conexus 1 (2018) 24-28

(C) 2018 Wolfgang Rother und Josette Baer. Dieser Beitrag darf im Rahmen der Lizenz CC BY-NC-ND 4.0 - Creative Commons: Namensnennung/ nicht kommerziell/keine Bearbeitungen - weiterverbreitet werden.

\section{https:// doi.org/10.24445/conexus.2018.01.004}

Prof. Dr. Wolfgang Rother, Universität Zürich, Philosophisches Seminar, Hirschengraben 56, 8001 Zürich

wolfgang.rother@philos.uzh.ch

Prof. Dr. Josette Baer, Universität Zürich, Philosophisches Seminar, Zürichbergstrasse 43, 8044 Zürich baer@philos.uzh.ch 